\title{
BMJ Open Epidural analgesia in critically ill patients with acute pancreatitis: the multicentre randomised controlled EPIPAN study protocol
}

Stéphanie Bulyez, ${ }^{1}$ Bruno Pereira, ${ }^{2}$ Elodie Caumon, ${ }^{2}$ Etienne Imhoff, ${ }^{1}$ Laurence Roszyk, ${ }^{3,4}$ Lise Bernard, ${ }^{5,6}$ Leo Bühler, ${ }^{7}$ Claudia Heidegger, ${ }^{8}$ Samir Jaber, ${ }^{9}$ Jean-Yves Lefrant, ${ }^{10}$ Russell Chabanne, ${ }^{1}$ Pierre-Marie Bertrand, ${ }^{11}$ Pierre-François Laterre, ${ }^{12}$ Philippe Guerci, ${ }^{13}$ Pierre-Eric Danin, ${ }^{14}$ Etienne Escudier, ${ }^{15}$ Achille Sossou, ${ }^{16}$ Dominique Morand, ${ }^{2}$ Vincent Sapin, ${ }^{3,4}$ Jean-Michel Constantin, ${ }^{1,4}$ Matthieu Jabaudon, ${ }^{1,4}$ on behalf of the EPIPAN study group and the AzuRea network

To cite: Bulyez S, Pereira B, Caumon $\mathrm{E}$, et al. Epidural analgesia in critically ill patients with acute pancreatitis: the multicentre randomised controlled EPIPAN study protocol. BMJ Open 2017;7:e015280. doi:10.1136/ bmjopen-2016-015280

- Prepublication history and additional material are published online only. To view please visit the journal online (http://dx.doi. org/10.1136/10.1136/bmjopen2016-015280).

Received 24 November 2016 Revised 26 January 2017 Accepted 6 March 2017

CrossMark

For numbered affiliations see end of article.

Correspondence to Dr Matthieu Jabaudon; mjabaudon@chuclermontferrand.fr

\section{ABSTRACT}

Background Acute pancreatitis (AP) is associated with high morbidity and mortality in its most severe forms. Most patients with severe AP require intubation and invasive mechanical ventilation, frequently for more than 7 days, which is associated with the worst outcome. Recent increasing evidence from preclinical and clinical studies support the beneficial effects of epidural analgesia (EA) in $A P$, such as increased gut barrier function and splanchnic, pancreatic and renal perfusion, decreased liver damage and inflammatory response, and reduced mortality. Because recent studies suggest that EA might be a safe procedure in the critically ill, we sought to determine whether EA reduced AP-associated respiratory failure and other major clinical outcomes in patients with AP. Methods and analysis The Epidural Analgesia for Pancreatitis (EPIPAN) trial is an investigator-initiated, prospective, multicentre, randomised controlled two-arm trial with assessor-blinded outcome assessment. The EPIPAN trial will randomise 148 patients with AP requiring admission to an intensive care unit (ICU) to receive EA (with patient-controlled epidural administration of ropivacaine and sufentanil) combined with standard care based on current recommendations on the treatment of AP (interventional group), or standard care alone (reference group). The primary outcome is the number of ventilatorfree days at day 30 . Secondary outcomes include main complications of AP (eg, organ failure and mortality, among others), levels of biological markers of systemic inflammation, epithelial lung injury, renal failure, and healthcare-associated costs.

Ethics and dissemination The study was approved by the appropriate ethics committee (CPP Sud-Est VI). Informed consent is required. If the combined application of EA and standard care proves superior to standard care alone in patients with $\mathrm{AP}$ in the ICU, the use of EA may become standard practice in experienced centres, thereby decreasing potential complications related to AP and its burden in critically ill patients. The results will be disseminated in a peer-reviewed journal.

\section{Strengths and limitations of this study}

- This is the first randomised controlled trial to investigate the effects of epidural analgesia (EA) on organ failure, mortality and clinical outcomes in critically ill patients with acute pancreatitis (AP) enrolled in a total of 11 French, Belgian and Swiss intensive care units.

- Other strengths are the inclusions performed around the clock, including nights and weekend, as routine clinical practice.

- In addition, our study includes the constitution of a biobank of plasma and urine sampled over the first week after inclusion, in order to assess the effects of EA on biological markers of inflammation, lung injury and renal failure.

- One limitation of the study is that the physicians are aware of the group of inclusion. However, assessors of study outcomes and biological measures are independent observers who do not know the group of inclusion.

- Another limitation may include poor generalisability of results from this study to inexperienced centres, because EA is a technique that is restricted to experienced anaesthetists and intensivists.

- Finally, some could highlight the potential risks associated with EA in critically ill patients with hyperinflammatory conditions such as AP, although previous studies have reported good feasibility and safety of EA in this setting. This trial will provide additional data on the safety of EA in ICU patients.

Trial registration number NCT02126332.

\section{INTRODUCTION}

Background and rationale

Acute pancreatitis (AP) is one of the most frequent gastrointestinal diseases, whose 
incidence in the USA has reached 35 per 100000 population annually. In 2009, AP was responsible for 275000 hospital admissions in the USA, with a total cost of over $\$ 2.5$ billion. $^{12}$ AP develops when intracellular protective mechanisms to prevent trypsinogen activation or reduce trypsin activity are overwhelmed. ${ }^{3}$ The initiating event may be any insult to the acinar cells that impairs the secretion of zymogen granules, such as alcohol abuse or gallstone migration into the common bile duct. Once the process of cellular injury is initiated, cellular membrane trafficking becomes chaotic, leading to the release of proinflammatory mediators (tumour necrosis factor (TNF)- $\alpha$, interleukin (IL) -6 , and IL-8). These mediators participate in increasing pancreatic vascular permeability that subsequently favours haemorrhage, oedema and eventually pancreatic necrosis. As these mediators are excreted into the circulation, systemic complications can arise, such as bacteraemia due to gut flora translocation, acute respiratory distress syndrome (ARDS), ${ }^{4}$ pleural effusions, gastrointestinal haemorrhage and renal failure. ${ }^{35-8}$

The revised Atlanta classification addresses the clinical course and severity of the disease. ${ }^{9}$ AP may be divided into two forms: interstitial oedematous pancreatitis, during the first week; and necrotising pancreatitis during a later phase (after 7 days). In approximately $80 \%$ of patients, the severity of AP is rather mild and resolves without serious morbidity. However, in up to $20 \%$ of patients, AP presents in a more severe form requiring admission to the intensive care unit (ICU) due to persistent organ failure. ${ }^{9} 10$ Mortality rates can reach $20-40 \%$ in severe AP because of multiorgan failure (MOF) and pancreatic necrosis. ${ }^{11}$

The amplifying effects of inflammatory and oxidative impairment often lead to severe AP-induced complications, which are often regarded as hallmarks of severe $\mathrm{AP}$ and herald poor outcome. In a recent French observational study of ICU patients with severe AP, $58 \%$ of patients developed acute respiratory failure requiring intubation and invasive mechanical ventilation (MV) (mean duration 15 days, SD 17 days), and such patients had higher mortality rates than those who were not intubated (34\% vs $1.4 \%) .{ }^{11}$ Since respiratory failure is the main cause of death in patients with severe AP, more work is needed to prevent and treat AP-associated respiratory failure. Despite recent substantial improvements in the multidisciplinary management of AP (eg, with regards to fluid therapy, intensive care management, prevention of infectious complications, nutritional support, biliary tract management or necrotising pancreatitis management), the prognosis of severe AP remains poor in patients who develop acute respiratory failure requiring intubation and invasive respiratory support. ${ }^{3912}$ Of note, available therapeutic approaches do not have a direct action on the pancreas itself but aim to attenuate the process of MOF present in the severe form of AP; no treatment of the cause(s) has been developed yet.

Epidural analgesia (EA) is one of the most widely utilised neural deafferentation techniques. It is used for analgesia during the perioperative period, but also for obstetrics labour and trauma as well as in the treatment of acute, chronic and cancer-related pain. ${ }^{13} 14$ Its objective is not only to block noxious afferent stimuli, but also to induce bilateral selective thoracic sympathetic blockade. In addition to analgesia itself, the modulatory effects of thoracic EA could improve organ perfusion with reduced complications in the perioperative period, thus possibly decreasing postoperative complications, shortening hospital stay and improving survival. ${ }^{14-16}$

EA has not yet been extensively assessed in the ICU setting in general, and in critically ill patients with severe AP in particular. Several studies suggest that thoracic EA might be a safe procedure in centres comprising anaesthetists with expertise in EA, and thoracic EA has already been used for years to treat pain during AP in critically ill patients in some centres. ${ }^{17-19}$ In addition, recent animal studies suggest that thoracic EA may decrease the severity of AP, with reduced respiratory, thromboembolic and abdominal complications. ${ }^{20-22}$ EA further decreased the severity of metabolic acidosis and tissue injury in animals, thus preventing the progression from oedematous to necrotising AP. ${ }^{23}$ EA may also restore pancreatic hypoperfusion induced by AP through blood flow redistribution from splanchnic to non-perfused pancreatic regions; ${ }^{24}{ }^{25}$ a recent clinical study suggests that EA could increase pancreatic arterial perfusion and improve clinical outcome in patients with AP. ${ }^{19}$ Findings from other experimental studies also support the beneficial effects of EA in severe AP, such as increased gut barrier function and renal perfusion, decreased liver damage and inflammatory response, and reduced mortality. 22242627

Despite such promising findings from preclinical studies, the effects of thoracic EA on major clinical outcomes have never been specifically assessed and its benefit in critically ill patients with AP remains uncertain.

\section{Objectives}

\section{Primary objective}

The primary objective of the study is to determine whether the use of thoracic EA combined with standard care is more effective at increasing ventilator-free days (VFD) at day 30 compared with standard care alone in critically ill patients with AP. The goal of the EPIPAN trial is therefore to test the impact of thoracic EA on respiratory failure, with the hypothesis that EA could influence survival and/ or the need for invasive MV and/or its duration when invasive MV is required.

\section{Secondary objectives}

The study will also aim to determine whether, in comparison with standard care alone, application of thoracic EA combined with standard care can improve survival, decrease major complications of AP (including sepsis and organ failure), reduce AP-related costs, reduce the need for medical, surgical and radiological interventions, and have an impact on the biological markers of systemic inflammation, lung injury and renal failure. 


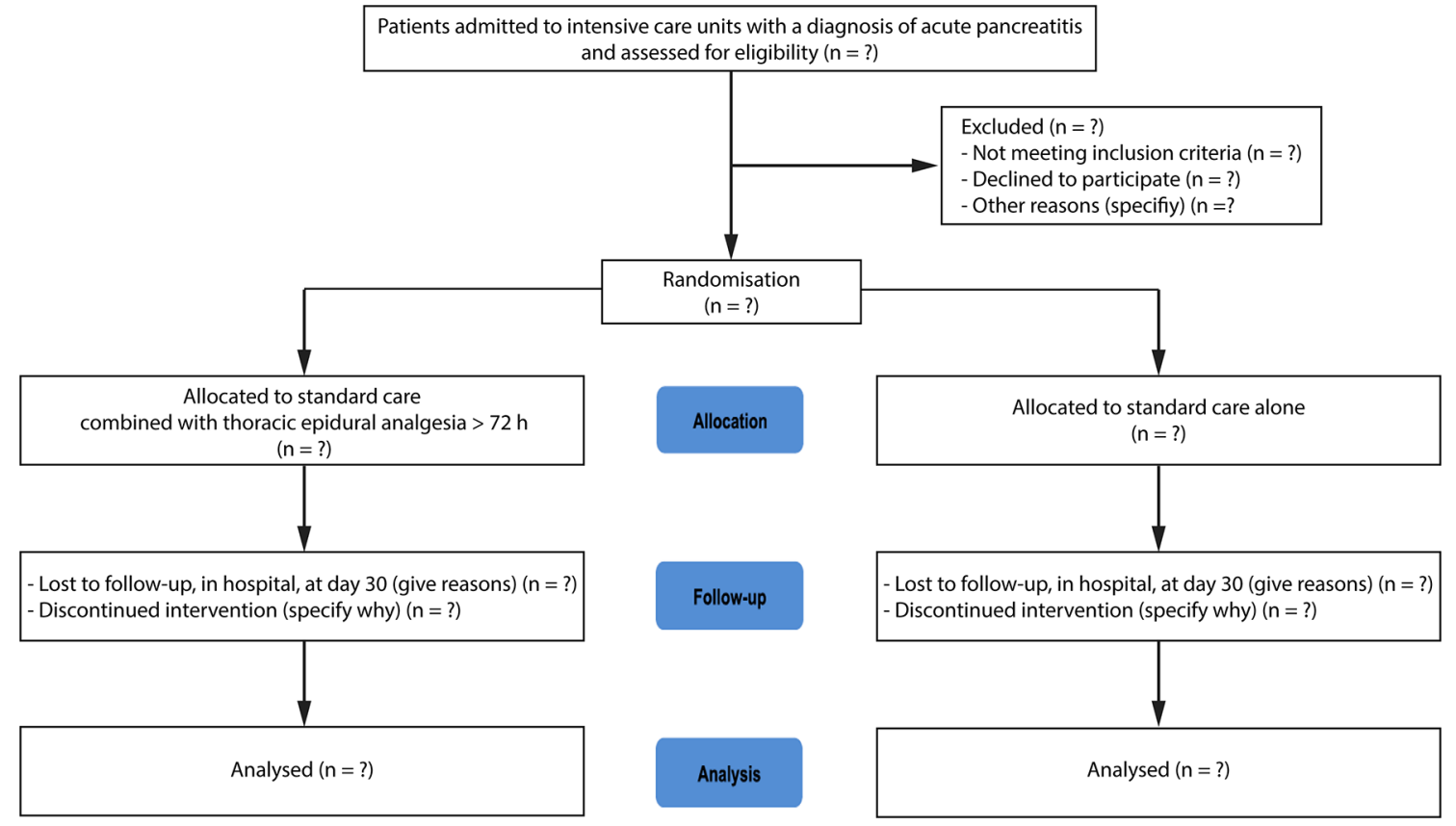

Figure 1 CONSORT diagram of the Epidural Analgesia for Pancreatitis (EPIPAN) trial illustrating the randomisation and flow of patients in the study.

\section{Trial design}

The Epidural Analgesia for Pancreatitis (EPIPAN) trial is an investigator-initiated, open-labelled, multicentre, randomised controlled two-arm trial.

\section{CONSORT diagram}

Figure 1 shows the CONSORT (Consolidated Standards of Reporting Trials) diagram of the EPIPAN trial.

\section{METHODS: PARTICIPANTS, INTERVENTIONS AND OUTCOMES}

This manuscript was written in accordance with the SPIRIT (Standard Protocol Items: Recommendations for Interventional Trials) guidelines (supporting file in the appendix). ${ }^{28}$

\section{Study setting}

The EPIPAN study involves a total of 11 mixed medical and surgical ICUs in France (Clermont-Ferrand (2 ICUs), Montpellier, Nîmes, Cannes, Nancy, Nice, Annecy, Le-Puyen-Velay), Belgium (Brussels) and Switzerland (Geneva).

\section{Eligibility criteria}

Inclusion criteria

Patients must be admitted to the ICU for AP, whatever the precise reason for admission (eg, pain management, organ failure).

\section{Exclusion criteria}

Patients fulfilling one or more of the following criteria are not included: age $<18$ years, pregnant or breastfeeding, protected person, known or suspected hypersensitivity to study drugs (ropivacaine and sufentanil are administered via the epidural catheter in the EA group, and epidural clonidine can be used as an iterative rescue treatment to achieve analgesia goals); also absolute contraindications to the placement of an epidural catheter: prothrombin time $<60 \%$, platelet count $<75 \times 10^{9} / \mathrm{L}$, curative anticoagulation unless it can be interrupted for at least 8 hours, local infection, active infection of the central nervous system, suspected or confirmed intracranial hypertension, history of back surgery including a dural space procedure, and refractory circulatory shock despite adequate resuscitation.

\section{Interventions}

Patients eligible for inclusion will be randomly assigned to the interventional group (EA combined with standard care) or to the reference group (standard care alone). Because the trial is primarily designed as a pragmatic trial, all patients will be managed by attending physicians as recommended in recent guidelines on the management of severe AP (standard care): early enteral nutrition when possible, resuscitation measures to correct hypovolaemia, maintenance of electrolyte balance, correction of acidosis, early diagnosis, and supportive treatment of complications. ${ }^{9} 12$ 29-31 In particular, criteria for intubation are based on current recommendations and include any of the following major clinical events: respiratory or cardiac arrest, respiratory pauses with loss of consciousness or gasping for air, massive aspiration, persistent inability to clear respiratory secretions, heart rate $<50$ / min with loss of alertness, and severe haemodynamic instability without response to fluid and vasoactive drugs. When invasive mechanical ventilation is needed, the use of a low-tidal-volume protective ventilatory strategy and recommendations on weaning from mechanical 
ventilation are strongly encouraged at each participating centre. $^{32}{ }^{33}$ Analgesia goals are the same in both groups, with regular evaluation of pain, at least every 4 hours. In conscious and communicating patients, a visual analogue score (VAS) for pain below 40/100 is targeted and a behavioural pain scale (BPS) of 3-4 is targeted in non-communicating patients. ${ }^{34}$ In both groups, a stepped multimodal approach to pain management will be applied based on routine protocols from each participating centre, combining opioid and non-opioid drugs \pm adjuvant drugs administered through the oral, enteral and/or intravenous routes, as recommended by the WHO's pain relief ladder. ${ }^{12} 36$

The interventional group will apply standard care combined with thoracic EA through an epidural catheter placed in an intervertebral space between the sixth and the ninth thoracic vertebra, with administration of a mixed solution of ropivacaine $(2 \mathrm{mg} / \mathrm{mL})$ and sufentanil $(0.5 \mu \mathrm{g} / \mathrm{mL})$, for at least 72 hours. In the study protocol, there is no strict time interval between ICU admission, enrolment in the study and placement of the epidural catheter. EA will be provided using a patient-controlled epidural analgesia (PCEA) device, with a continuous infusion rate of $5-15 \mathrm{~mL} /$ hour and a bolus of $3-10 \mathrm{~mL}$ every 10 min maximum. If the patient is not able to self-administer EA, nurses are encouraged to administer boluses to achieve analgesia goals if necessary-for example, before possibly painful nursing procedures. In addition, iterative epidural administrations of clonidine $(1 \mu \mathrm{g} / \mathrm{kg})$ may be used by attending physicians to achieve analgesia goals. ${ }^{37}$ The drugs used during EA in this trial will be provided in an unblinded manner by the Department of Pharmacy at Clermont-Ferrand university hospital to all participating centres.

Because of insufficient evidence regarding the optimal duration of EA in ICU patients, ${ }^{17} 1838$ the total duration of EA will be chosen by participating physicians for each patient, given that it has been administered for at least 72 hours. Weaning of EA and removal of the epidural catheter will be conducted according to the recommendations and routine protocols of each participating centre.

\section{Outcomes}

\section{Primary outcome measures}

The primary outcome variable is the number of VFD at day 30 , defined as the number of days from day 0 (inclusion) to day 30 after inclusion on which a patient is able to breathe without invasive assistance. A difference in VFD can reflect a difference in mortality, ventilator days, or both.

\section{Secondary outcome measures}

Secondary outcomes are: the need for and duration of invasive and/or non-invasive MV at day 30; the incidence of AP-related complications at day 30 (death, organ failure, severe sepsis, septic shock, ${ }^{32}$ ARDS, ${ }^{4}$ acute respiratory failure, abdominal compartment syndrome, intra- or extra-abdominal sepsis, pancreatic necrosis or abscess (infected or not), haemodynamic failure requiring vasopressor therapy, acute kidney injury, ${ }^{39}$ requirement for renal replacement therapy, infected intra-abdominal abscesses requiring drainage (radiological, endoscopic or surgical), intolerance to enteral feeding); analgesia scores (VAS, BPS); need for sedation (drugs, doses, level of sedation using the Richmond Agitation-Sedation Scale); ${ }^{40}{ }^{41}$ lengths of stay in the ICU and in hospital; the need for ICU readmission within 30 days after inclusion; levels on days 0,2 and 7 after inclusion of biological markers (as assessed in duplicate using commercially available kits) of systemic inflammation (plasma levels of IL-6) $;^{42}$ lung epithelial injury (plasma levels of the soluble form of the receptor for advanced glycation end-products, sRAGE) ${ }^{43} 44$ and acute kidney injury (plasma levels of neutrophil gelatinase-associated lipocalin (NGAL); ${ }^{45} 46$ urine levels of tissue inhibitor of metalloproteinase 2 (TIMP-2) and insulin-like growth factor binding protein 7 (IGFBP-7) $;{ }^{47}$ and healthcare-related costs at day 30 . The need for antibiotic or antifungal therapy will be assessed. Any minor or major complication (eg, epidural haematoma or infection) that could be attributable to $\mathrm{EA}$ and/or the epidural catheter will also be rigorously documented.

\section{Participant timeline}

The participant timeline is described in table 1 .

\section{Recruitment}

Patients are expected to be included during a 3-year inclusion period that began in June 2014. This duration was estimated based on the number of admissions for AP at each participating centre during a 5-year period (20092014).

2013-2014: Protocol, approvals from the ethics committee (CPP Sud-Est VI) and the French Medicine agency (Agence Nationale de Sécurité du Médicament, ANSM); trial tool development (case report form, randomisation system).

2014-2017: Inclusion of patients.

2017: Cleaning and closure of the database. Data analyses, writing of the manuscript and submission for publication.

A prolongation of the inclusion period will be requested if needed based on observed inclusion rate.

\section{METHODS: ASSIGNMENT OF INTERVENTIONS Allocation and sequence generation}

An electronic, centralised web-based data management system will be used for randomisation (TENALEA, FormsVision BV, the Netherlands). To minimise selection bias, randomisation will be performed in strict sequence-that is, when a subject is confirmed as eligible for randomisation, the next unassigned randomisation number in sequence will be given by the TENALEA system. Randomisation will be stratified and minimised based on the recruiting centre, the duration of symptoms (either more or less than 48 hours from first symptoms, 


\section{Inclusion}

$\begin{array}{lllllllll}\text { (day 0) Day } 1 & \text { Day } 2 & \text { Day } 3 & \text { Day } 4 & \text { Day } 5 & \text { Day } 6 & \text { Day } 7 & \text { Day } 15 & \text { Day } 30\end{array}$

\begin{tabular}{ll}
\hline Informed consent & $\mathrm{X}$ \\
Eligibility: check inclusion and & $\mathrm{X}$ \\
exclusion criteria &
\end{tabular}

\begin{tabular}{|c|c|c|c|c|c|c|c|c|c|c|}
\hline Randomisation & $x$ & & & & & & & & & \\
\hline $\begin{array}{l}\text { Filling of case report forms } \\
\text { (including data on } E A \text { in the } \\
\text { interventional group) }\end{array}$ & $x$ & $x$ & $x$ & $x$ & $x$ & $x$ & $x$ & $x$ & $x$ & $\mathrm{x}$ \\
\hline $\begin{array}{l}\text { Sampling of blood and urine } \\
\text { specimens }\end{array}$ & $x$ & & $x$ & & & & & $x$ & & \\
\hline $\begin{array}{l}\text { Complications of acute pancreatitis } \\
\text { and survival status }\end{array}$ & & & & & & & & & $x$ & $\mathrm{x}$ \\
\hline End of study & & & & & & & & & & $\mathrm{x}$ \\
\hline
\end{tabular}

eg, abdominal pain, to inclusion) and severity of AP as assessed by the modified Marshall scoring system for organ dysfunction. ${ }^{9} 48$ This scoring system has the merit of simplicity, universal applicability across international centres, and the ability to stratify disease severity easily and objectively based on respiratory, renal and/or haemodynamic failure. ${ }^{49}$ A score of 2 or more usually defines the presence of organ failure, and three strata of severity (scores equal to $0,1-2$ or 3-4) are used to stratify randomisation on the degree of organ failure in the EPIPAN trial.

\section{Blinding}

This is an open-label, unblinded trial for patients and the physicians in charge of the patients, because of the nature of the intervention (placement and maintenance of EA through an epidural catheter). Although some systems may be proposed to ensure at least partial blinding to the patient when EA is assessed, ${ }^{50}$ such systems were not included in the trial design in order to ensure better feasibility among multiple centres. However, assessors of clinical and biological data in charge of statistical analyses and outcome assessment will be masked as to the subjects' assigned group.

\section{METHODS: DATA COLLECTION, MANAGEMENT AND ANALYSIS}

\section{Data collection and management}

Study data are prospectively collected and managed by trained research coordinators and/or investigators from each participating centre, using REDCap electronic data capture tools hosted at Clermont-Ferrand university hospital. ${ }^{51}$ REDCap (Research Electronic Data Capture) is a secure, web-based application designed to support data capture for research studies, providing: (1) an intuitive interface for validated data entry; (2) audit trails for tracking data manipulation and export procedures; (3) automated export procedures for seamless data downloads to common statistical packages; and (4) procedures for importing data from external sources.

The following data are collected and registered at ICU admission and on inclusion: baseline demographics and characteristics (age, sex, weight, height, body temperature, delay between the onset of AP and ICU admission/study inclusion, comorbidities and coexisting conditions), baseline severity of illness (modified Marshall scoring system, Simplified Acute Physiologic Score (SAPS) II, Sequential Organ Failure Assessment (SOFA)), usual clinical and biological variables that are measured in critically ill patients, organ failure, and treatments. From inclusion to day 30 the following will be assessed: survival status; main complications of AP (eg, organ failure, sepsis); the need for therapeutic interventions (such as surgery or endoscopic manoeuvres; MV (either invasive or non-invasive); vasopressor support; continuous renal replacement therapy and/or antibiotic therapy; duration of MV if required; and length of stay in the ICU/hospital. Biological samples will be collected in each participating centre, before shipment of all samples to the Department of Medical Biochemistry and Molecular Biology at Clermont-Ferrand university hospital for blinded measurements.

\section{Statistical methods}

\section{Sample size estimation}

According to previous studies from the literature, ${ }^{11} 19$ we have estimated that a sample size of $n=74$ patients per group would provide $80 \%$ statistical power to detect an absolute between-group difference of 7 days (with an SD of \pm 15 ) in the primary outcome-that is, in the number of VFD at day 30 after randomization (expected number of VFD at day 30: $20 \pm 15$ vs $13 \pm 15$ ) -for a two-sided type I error of $5 \%$.

Given theoretical concerns related to possible adverse effects of EA in ICU patients, an interim safety analysis will be performed after data for 74 patients have been collected. The independent Data and Safety Monitoring 
Board (DSMB) will recommend that the trial be stopped if it is found that the conduct of the trial compromises patient safety (a between-group difference in mortality or VFD at day 30).

\section{Statistical analysis}

A predefined statistical analysis plan will be followed. Statistical analyses will be conducted using Stata software (version 14, StataCorp, College Station, USA). A two-sided $p$ value $<0.05$ will be considered to indicate statistical significance.

Concerning the primary outcome, the comparison between interventional and reference groups will be analysed using Student's t-test or the Mann-Whitney test if assumptions of the t-test are not met. Normality will be studied by the Shapiro-Wilk test and homoscedasticity using the Fisher-Snedecor test. Results will be expressed as effect-sizes and 95\% confidence intervals (95\% CI). Intention-to-treat (ITT) analysis of data from all randomised patients (except patients who withdraw their consent and those who do not meet the inclusion criteria), including those from the interventional group who did not receive EA for at least 72 hours, will be considered for the primary analysis. Then, the analysis of the primary outcome will be completed by multivariate analysis using a linear mixed model to take into account: (1) fixed effects covariates determined according to univariate results and to clinical relevance (duration of symptoms (either more or less than 48 hours from first symptoms, eg, abdominal pain, to inclusion) and severity of AP as assessed by the modified Marshall scoring system for organ dysfunction); and (2) centre as random-effects (to measure between and within centre variability). The normality of residuals will be studied as described previously. Results will be expressed as regression coefficients and $95 \% \mathrm{CI}$.

Other continuous endpoints (eg, level of sedation using the Richmond Agitation-Sedation Scale, analgesia scores, doses of drugs, length of stay in ICU/hospital, levels and kinetics of biological markers, duration of MV, and healthcare-related costs at day 30 ) will be analysed in the same way.

Categorical parameters (death, organ failure, severe sepsis, septic shock, ARDS, the need for MV, acute respiratory failure, abdominal compartment syndrome, intra- or extra-abdominal sepsis, pancreas necrosis (infected or not) as assessed by CT, haemodynamic failure requiring vasopressor support, acute kidney injury, the need for renal replacement therapy, intra-abdominal collection requiring radiological, surgical or endoscopic drainage) will be analysed using $\chi^{2}$ or Fisher's exact tests for univariate analysis, and generalised linear mixed model (logistic for dichotomous dependent endpoint or Poisson if more appropriate) for multivariate analysis. Type I error will be adjusted using the Hochberg method if appropriate. Results will be expressed as relative risks and 95\% CI. These data will also be analysed as censored data, when appropriate; survival analyses will be performed with the
Kaplan-Meier estimator and differences between groups will then be assessed using the log-rank test. The assumption of log-linearity of risk and the proportional hazards will be checked beforehand. Results will be expressed as hazard ratios and $95 \%$ CI. The tolerance of enteral nutrition and/or the incidence of signs of gastrointestinal intolerance (nausea, vomiting, and ileus) will be analysed similarly.

Longitudinal analyses of repeated measures (levels on days 0,2 and 7 after inclusion of biological markers of systemic inflammation, lung epithelial injury and acute kidney injury) will be studied using random-effect models (linear or generalised linear), to take into account patients as random-effect (slope and intercept), nestled in centre random-effect.

According to clinical relevance and to CONSORT recommendations, subgroup analyses depending on the presence or absence of epidural analgesia will be proposed after the study of subgroup $\mathrm{x}$ randomisation group interaction in regression models.

Per-protocol analyses will also be conducted after intention-to-treat analysis is performed. Results from per-protocol analyses will be compared with those from intention-to-treat analyses. A particular focus will be given to safety and patients who are lost to follow-up. A sensitivity analysis will be performed and the nature of missing data will be studied (missing at random or not). According to this study, the most appropriate approach to the imputation of missing data will be proposed (maximum bias (eg, last observation carried forward vs. baseline observation carried forward) or estimation proposed by Verbeke and Molenberghs for repeated data).

\section{METHODS: MONITORING}

\section{Data monitoring}

Before the start of patient recruitment, all physicians and other healthcare workers in the ICU attended formal training sessions on the study protocol and data collection.

The physicians, clinical research nurses and/or clinical research assistants are in charge of daily patient screening and inclusion, ensuring compliance with the study protocol and collecting the study data. Patients who are admitted to the ICU with AP but who are not included, and the reasons why they are not included, will be recorded anonymously into a screening log in each centre.

Data monitoring and quality control will be conducted at least annually in all participating centres by official representatives from the study promoter-that is, from the Department of Clinical Research and Innovation at Clermont-Ferrand university hospital.

\section{Harms}

The trial may be temporarily stopped for an individual patient, at the discretion of the attending physician, in 
the case of major serious adverse events suspected to be associated with EA.

Given potential theoretical concerns related to the possible adverse effects of EA in ICU patients, an interim safety analysis will be performed after data for 74 patients have been obtained using the Lan and DeMets method (East software, Cytel Inc, Cambridge, MA, USA). The independent Data and Safety Monitoring Board (DSMB) will recommend that the trial be stopped if it is found that the conduct of the trial compromises patient safety (a between-group difference in mortality or VFD at day 30$)$.

All adverse events thought to be related to the trial will be reported to the trial coordinating centre. According to the French Public Health Code, all suspected unexpected serious adverse events will be reported to the ANSM. In addition, this information will be submitted to the DSMB.

\section{Auditing}

An independent DSMB, composed of three experts (Professors Hervé Dupont, Thomas Lescot and Philippe Montravers) will monitor the safety of the trial. The DSMB will be responsible for safeguarding the interests of trial participants, for assessing the safety and efficacy of the interventions during the trial, and for monitoring the overall conduct of the clinical trial. To contribute to enhancing the integrity of the trial, the DSMB may also formulate recommendations relating to the recruitment/ retention of participants, their management, improving adherence to protocol-specified regimens and retention of participants, and the procedures for data management and quality control.

\section{ETHICS AND DISSEMINATION \\ Research ethics approval}

The EPIPAN study is conducted in accordance with the Declaration of Helsinki and was registered at http:/ / www. clinicaltrial.gov on 11 April 2014 with trial identification number NCT02126332. The trial was approved by the ethics committee CPP Sud-Est VI in June 2014 (approval number AU1090) and ANSM (approval number 131557A-32) in January 2014. Approvals from appropriate authorities were also obtained for Belgian and Swiss centres. Any change to eligibility criteria, outcomes and analyses will be communicated to investigators, the ethics committee and the ANSM to obtain their approval.

\section{Consent or assent}

Three methods of consent will be used, as required by the Institutional Review Board in accordance with the 2013 Declaration of Helsinki. Whenever possible, the patient will be included after written informed consent. However, the patient may be unable to provide informed consent because of the severity of illness (eg, altered mental status, use of sedation). These patients will be included after written informed consent is provided by the next of kin, or using an emergency procedure (investigator signature, countersigned by an independent physician) if the next of kin is not present. When available, and as soon as possible after recovery, patients will be retrospectively asked for written consent to continue the trial.

\section{Confidentiality}

Data will be handled in a confidential and anonymous manner, according to French law. All original records will be archived at trial sites for 15 years. The clean database file will be anonymised and kept for 15 years.

\section{Declaration of interest}

The study is an investigator-initiated trial. Study promotion is performed by Clermont-Ferrand university hospital, Clermont-Ferrand, France. There is no industry support or involvement in the trial. The principal investigators have no financial or other competing interests.

\section{Funding}

This trial is supported by grants from the Société Française d'Anesthésie et de Réanimation (Contrat de Recherche SFAR 2015) and from Clermont-Ferrand university hospital (Appel d'Offre Interne 2014, CHU Clermont-Ferrand). The funders have no influence on the study design, conduct and analysis or in the preparation of this article.

\section{Dissemination policy}

Findings will be published in peer-reviewed journals and presented at local, national and international meetings and conferences to publicise and explain the research to clinicians, commissioners and service users. All investigators will have access to the final dataset. Participant-level datasets will be made accessible on a controlled access basis.

\section{DISCUSSION}

Severe acute pancreatitis requiring ICU admission is associated with high morbidity and mortality, especially in patients who need intubation and invasive ventilation. ${ }^{9} 11$ Optimising the management of critically ill patients with AP is therefore of particular importance, especially in those with, or at risk of, acute respiratory failure requiring intubation/prolonged ventilation, death, or both. However, and despite recent improvements in ICU practice in general, current guidelines on the management of severe AP only include supportive measures such as early enteral nutrition, haemodynamic resuscitation, maintenance of electrolyte balance, correction of acidosis, and early diagnosis and treatment of complications (eg, with appropriate use of anti-infectious drugs, radiologic drainage, endoscopic manoeuvres and/or elective surgery in selected patients) $.^{912} 29-31$

EA is primarily an analgesic technique that is used by anaesthetists to treat pain in the perioperative period, for obstetrical analgesia, and after severe chest trauma. ${ }^{52}$ There has been recent interest in the use of EA as treatment for AP, and growing evidence from experimental studies now support beneficial effects of EA that include augmented ileal mucosal capillary perfusion, restored 
pancreatic microcirculation, increased gut barrier function and renal perfusion, decreased severity, and improved survival. ${ }^{20-22} 24262753$ However, only one small recent randomised pilot study in 35 patients with $\mathrm{AP}$ was found to translate such promising preclinical findings into the clinical settings. ${ }^{19}$ In this study, the median duration of EA was 5.7 days, and no complications of the epidural procedure were reported. An improvement in perfusion of the pancreas was observed in $43 \%$ of measurements in the EA group versus $7 \%$ in the control group ( $\mathrm{p}=0.0025)$; however, although analgesia was better when EA was used, there was no significant between-group differences in other clinical outcomes (eg, the need for necrosectomy, length of stay in hospital and mortality), probably due to a lack of statistical power. ${ }^{19}$ The EPIPAN trial is the first randomised controlled study powered to investigate the effectiveness of thoracic EA combined with standard care on major clinical outcomes in critically ill patients with EA, with specific emphasis on respiratory outcomes and survival. ${ }^{20}$

This study may have some limitations. First, no strict definition for severe AP is used to enrol patients. Instead, all patients admitted to the ICU with AP are eligible whatever the precise reason for admission (eg, pain management, development of organ failure). However, we believe that randomisation, as stratified on the modified Marshall scoring system (thus distinguishing patients with absent, moderate and severe organ failure), among other parameters, should ensure similar distribution of the severity of AP in both arms. Second, we acknowledge that the EPIPAN trial does not include precise (sub) protocols addressing every single aspect of the management of patients with AP (eg, enteral feeding, its initiation, route of administration and dose), because it was believed that this would have hampered the inclusion of patients and the feasibility of this pragmatic study. Instead, current guidelines for the management of AP are actively encouraged among study participants. ${ }^{9}{ }^{12} 29-31$ Although the implementation of consensual recommendations will not be specifically assessed while the study is still ongoing, and as it may have an impact on the findings and their interpretation, adherence of physicians from participating centres to these guidelines will be analysed after study completion. Third, this trial, whatever its results, will not address the question of the selection of patients with AP who may best benefit of EA. However, analyses of clinical and biological subphenotypes of patients included in the trial, and their responses to EA, should possibly inform on how to better select patients for future studies. Fourth, another limitation may include the limited generalisability of the results obtained from this study because EA is a technique that is restricted to experienced anaesthetists and intensivists. Fifth, the expected between-group difference in the primary endpoint, as extrapolated from the study from Jung $e t a l,{ }^{11}$ may be debatable and considered as too optimistic. Although we acknowledge that this choice is debatable, we believe that it is an acceptable compromise between study feasibility and clinical relevance, while ensuring the building of the largest cohort of critically ill patients with AP to date. Finally, some could highlight the potential risks associated with EA in critically ill patients with hyperinflammatory conditions such as $\mathrm{AP}^{385455}$ although previous studies suggest good feasibility and safety of EA in this setting. Findings from the EPIPAN trial will undoubtedly provide new data on both the efficacy and the safety of EA during clinical AP.

This study also has several strengths. First, it is to our knowledge the largest randomised controlled trial in critically ill patients with AP. Even in the case of 'negative' results, data from this trial will contribute to a better understanding of the characteristics, management and prognosis of ICU patients with AP. Second, it is the first trial powered to assess specifically the effects of EA on major patient outcomes such as respiratory outcomes and 30-day mortality. In addition, other strengths are the inclusions performed around the clock, with nights and weekends included as routine clinical practice. Third, this study includes the constitution of a biobank of plasma and urine sampled over the first week after inclusion, in order to assess biological markers of inflammation, lung injury and renal failure and the effects of EA on such markers. Finally, and despite an open-label design, one further strength of the study is that the final assessors of the clinical and biological data, who will be in charge of statistical analyses and outcome assessment, remain masked as to the subjects' assigned group, thus limiting bias.

In conclusion, the EPIPAN trial is an investigator-initiated, pragmatic, multicentre, randomised controlled trial powered to test the hypothesis that adding thoracic EA to standard care in comparison to standard care alone may improve respiratory outcomes-that is, increase the number of ventilator-free days at day 30-in critically ill patients with AP. The EPIPAN trial will also assess the effects of combined EA and standard care on the main complications of AP and other major patient outcomes.

\section{Author affiliations}

${ }^{1}$ Department of Perioperative Medicine, CHU Clermont-Ferrand, Clermont-Ferrand, France

${ }^{2}$ Department of Clinical Research and Innovation (DRCI), CHU Clermont-Ferrand, Clermont-Ferrand, France

${ }^{3}$ Department of Medical Biochemistry and Molecular Biology, CHU Clermont-

Ferrand, Clermont-Ferrand, France

${ }^{4}$ Université Clermont Auvergne, CNRS UMR 6293, INSERM U1103, GReD, ClermontFerrand, France

${ }^{5}$ Department of Pharmacy, CHU Clermont-Ferrand, Clermont-Ferrand, France ${ }^{6}$ Université Clermont Auvergne, Clermont-Ferrand, France

${ }^{7}$ Department of Surgery, Geneva university hospitals, Geneva, Switzerland

${ }^{8}$ Division of Intensive Care, Geneva university hospitals, Geneva, Switzerland ${ }^{9}$ Department of Anesthesiology and Critical Care Medicine B (DAR B), Saint-Eloi teaching hospital, Montpellier university hospital, Montpellier, France

${ }^{10}$ Division of Anesthesiology, Critical Care, Pain and Emergency Medicine, Nîmes university hospital and EA 2992, Université Montpellier, Nîmes, France

${ }^{11}$ Intensive Care Unit, Cannes general hospital, Cannes, France

${ }^{12}$ Department of Critical Care Medicine, Saint Luc university hospital, Université Catholique de Louvain, Brussels, Belgium 
${ }^{13}$ Department of Anesthesiology and Intensive Care Medicine, Nancy university hospital, Nancy, France

${ }^{14}$ Anesthesia and Surgical Intensive Care, Nice Archet 2 university hospital and INSERM U1065, team 8, Nice, France

${ }^{15}$ Intensive Care Unit, Annecy Genevois general hospital, Annecy, France

${ }^{16}$ Department of Anesthesiology and Critical Care Medicine, Emile-Roux general hospital, Le Puy-en-Velay, France

Contributors MJ takes responsibility for the content of the manuscript. MJ, SB, EI, $B P, E C, D M$ and JMC were involved in the conception, hypotheses delineation, and design of the study, acquisition and analysis of the data, in writing the article and in its revision prior to submission. All other authors were involved in the design of the study, acquisition, and analysis of the data, in writing the article and in its revision prior to submission.

Competing interests None declared.

Ethics approval Comité de Protection des Personnes (CPP) Sud-Est VI.

Provenance and peer review Not commissioned; externally peer reviewed.

Open Access This is an Open Access article distributed in accordance with the Creative Commons Attribution Non Commercial (CC BY-NC 4.0) license, which permits others to distribute, remix, adapt, build upon this work non-commercially, and license their derivative works on different terms, provided the original work is properly cited and the use is non-commercial. See: http://creativecommons.org/ licenses/by-nc/4.0/

(C) Article author(s) (or their employer(s) unless otherwise stated in the text of the article) 2017. All rights reserved. No commercial use is permitted unless otherwise expressly granted.

\section{REFERENCES}

1. Peery AF, Dellon ES, Lund J, et al. Burden of gastrointestinal disease in the United States: 2012 update. Gastroenterology 2012;143:1179-87.

2. Swaroop VS, Chari ST, Clain JE. Severe acute pancreatitis. JAMA 2004;291:2865-8.

3. Lankisch PG, Apte M, Banks PA. Acute pancreatitis. Lancet 2015;386:85-96

4. Acute respiratory distress syndrome: the Berlin definition. JAMA 2012;307.

5. Whitcomb DC. Clinical practice. acute pancreatitis. N Engl J Med 2006;354:2142-50.

6. Baron TH, Morgan DE. Acute necrotizing pancreatitis. N Engl J Med 1999;340:1412-7.

7. Dombernowsky T, Kristensen MØ, Rysgaard S, et al. Risk factors for and impact of respiratory failure on mortality in the early phase of acute pancreatitis. Pancreatology 2016;16:756-60.

8. Klar E, Schratt W, Foitzik T, et al. Impact of microcirculatory flow pattern changes on the development of acute edematous and necrotizing pancreatitis in rabbit pancreas. Dig Dis Sci 1994:39:2639-44.

9. Banks PA, Bollen TL, Dervenis C, et al. Classification of acute pancreatitis--2012: revision of the Atlanta classification and definitions by international consensus. Gut 2013;62:102-11.

10. Lund $\mathrm{H}$, Tønnesen $\mathrm{H}$, Tønnesen $\mathrm{MH}$, et al. Long-term recurrence and death rates after acute pancreatitis. Scand J Gastroenterol 2006;41:234-8.

11. Jung B, Carr J, Chanques G, et al. [Severe and acute pancreatitis admitted in intensive care: a prospective epidemiological multiple centre study using CClin network database]. Ann Fr Anesth Reanim 2011;30:105-12.

12. Tenner S, Baillie J, DeWitt J, et al. American College of Gastroenterology guideline: management of acute pancreatitis. Am $J$ Gastroenterol 2013:108:14001416-1415.

13. Clemente A, Carli F. The physiological effects of thoracic epidural anesthesia and analgesia on the cardiovascular, respiratory and gastrointestinal systems. Minerva Anestesiol 2008;74:549-63.

14. Bardia A, Sood A, Mahmood F, et al. Combined epidural-general anesthesia vs general anesthesia alone for elective abdominal aortic aneurysm repair. JAMA Surg 2016;151:1116.

15. Von Dossow V, Welte M, Zaune U, et al. Thoracic epidural anesthesia combined with general anesthesia: the preferred anesthetic technique for thoracic surgery. Anesth Analg 2001;92:848-54.

16. Rodgers A, Walker N, Schug S, et al. Reduction of postoperative mortality and morbidity with epidural or spinal anaesthesia: results from overview of randomised trials. BMJ 2000;321:1493.
17. Jabaudon $\mathrm{M}$, Chabanne R, Sossou A, et al. Epidural analgesia in the intensive care unit: an observational series of 121 patients. Anaesth Crit Care Pain Med 2015;34:217-23.

18. Bernhardt A, Kortgen A, Niesel HCh, et al. [Using epidural anesthesia in patients with acute pancreatitis--prospective study of 121 patients]. Anaesthesiol Reanim 2002;27:16-22.

19. Sadowski SM, Andres A, Morel P, et al. Epidural anesthesia improves pancreatic perfusion and decreases the severity of acute pancreatitis. World J Gastroenterol 2015;21:12448-56.

20. Windisch O, Heidegger CP, Giraud R, et al. Thoracic epidural analgesia: a new approach for the treatment of acute pancreatitis? Crit Care 2016;20:116.

21. Demirag A, Pastor CM, Morel P, et al. Epidural anaesthesia restores pancreatic microcirculation and decreases the severity of acute pancreatitis. World J Gastroenterol 2006;12:915-20.

22. Bachmann KA, Trepte CJ, Tomkötter L, et al. Effects of thoracic epidural anesthesia on survival and microcirculation in severe acute pancreatitis: a randomized experimental trial. Crit Care 2013;17:R281

23. Ai K, Kotake $\mathrm{Y}$, Satoh $\mathrm{T}$, et al. Epidural anesthesia retards intestinal acidosis and reduces portal vein endotoxin concentrations during progressive hypoxia in rabbits. Anesthesiology 2001;94:263-9.

24. Freise $\mathrm{H}$, Lauer $\mathrm{S}$, Anthonsen $\mathrm{S}$, et al. Thoracic epidural analgesia augments ileal mucosal capillary perfusion and improves survival in severe acute pancreatitis in rats. Anesthesiology 2006;105:354-9.

25. Freise $\mathrm{H}$, Lauer S, Konietzny E, et al. Hepatic effects of thoracic epidural analgesia in experimental severe acute pancreatitis. Anesthesiology 2009:111:1249-56.

26. Enigk F, Wagner A, Samapati $R$, et al. Thoracic epidural anesthesia decreases endotoxin-induced endothelial injury. BMC Anesthesiol 2014:14:23.

27. Schäper J, Wagner A, Enigk F, et al. Regional sympathetic blockade attenuates activation of intestinal macrophages and reduces gut barrier failure. Anesthesiology 2013;118:134-42.

28. Chan AW, Tetzlaff JM, Gøtzsche PC, et al. SPIRIT 2013 explanation and elaboration: guidance for protocols of clinical trials. BMJ 2013;346:e7586.

29. Greenberg JA, Hsu J, Bawazeer M, et al. Clinical practice guideline: management of acute pancreatitis. Can J Surg 2016;59:128-40.

30. Zerem E. Treatment of severe acute pancreatitis and its complications. World J Gastroenterol 2014;20:13879-92.

31. Working Group IAP/APA acute pancreatitis guidelines. IAP/APA evidence-based guidelines for the management of acute pancreatitis. Pancreatology 2013;13:e1-15.

32. Dellinger RP, Levy MM, Rhodes A, et al. Surviving sepsis campaign: international guidelines for management of severe sepsis and septic shock: 2012. Crit Care Med 2013;41:580-637.

33. Boles JM, Bion J, Connors A, et al. Weaning from mechanical ventilation. Eur Respir J 2007;29:1033-56.

34. Payen JF, Bru O, Bosson JL, et al. Assessing pain in critically ill sedated patients by using a behavioral pain scale. Crit Care Med 2001;29:2258-63.

35. Aïssaoui Y, Zeggwagh AA, Zekraoui A, et al. Validation of a behavioral pain scale in critically ill, sedated, and mechanically ventilated patients. Anesth Analg 2005;101:1470-6.

36. WHO's cancer pain ladder for adults. 2013. http://www.who.int/ cancer/palliative/painladder/en/ (accessed 6 Oct 2016).

37. Wu CL, Cohen SR, Richman JM, et al. Efficacy of postoperative patient-controlled and continuous infusion epidural analgesia versus intravenous patient-controlled analgesia with opioids: a metaanalysis. Anesthesiology 2005;103:1079-88.

38. Figueiredo S, Benhamou D. Epidural analgesia in ICU: useful and effective probably, safe maybe. Anaesth Crit Care Pain Med 2015;34:185-6.

39. Kellum JA, Lameire N; KDIGO AKI Guideline Work Group. Diagnosis, evaluation, and management of acute kidney injury: a KDIGO summary (Part 1). Crit Care 2013;17:204.

40. Sessler CN, Gosnell MS, Grap MJ, et al. The Richmond agitationsedation scale: validity and reliability in adult intensive care unit patients. Am J Respir Crit Care Med 2002;166:1338-44.

41. Ely EW, Truman B, Shintani A, et al. Monitoring sedation status over time in ICU patients: reliability and validity of the Richmond AgitationSedation Scale (RASS). JAMA 2003;289:2983-91.

42. Zhang $\mathrm{H}$, Neuhöfer P, Song L, et al. IL-6 trans-signaling promotes pancreatitis-associated lung injury and lethality. J Clin Invest 2013:123:1019-31.

43. Uchida T, Shirasawa M, Ware LB, et al. Receptor for advanced glycation end-products is a marker of type I cell injury in acute lung injury. Am J Respir Crit Care Med 2006;173:1008-15.

44. Jabaudon M, Blondonnet R, Roszyk L, et al. Soluble receptor for advanced glycation end-products predicts impaired alveolar fluid 
clearance in acute respiratory distress syndrome. Am J Respir Crit Care Med 2015;192:191-9.

45. Haase M, Bellomo R, Devarajan P, et al. Accuracy of neutrophil gelatinase-associated lipocalin (NGAL) in diagnosis and prognosis in acute kidney injury: a systematic review and meta-analysis. $\mathrm{Am} \mathrm{J}$ Kidney Dis 2009;54:1012-24.

46. Constantin JM, Futier E, Perbet S, et al. Plasma neutrophil gelatinase-associated lipocalin is an early marker of acute kidney injury in adult critically ill patients: a prospective study. J Crit Care 2010;25:176.e1-176.e6

47. Vijayan A, Faubel S, Askenazi DJ, et al. Clinical use of the urine biomarker [TIMP-2] $\times$ [IGFBP7] for acute kidney injury risk assessment. Am J Kidney Dis 2016;68:19-28.

48. Marshall JC, Cook DJ, Christou NV, et al. Multiple organ dysfunction score: a reliable descriptor of a complex clinical outcome. Crit Care Med 1995;23:1638-52.

49. Working Party of the British Society of Gastroenterology, Association of Surgeons of Great Britain and Ireland, Pancreatic Society of Great Britain and Ireland, Association of Upper GI Surgeons of Great Britain and Ireland. UK guidelines for the management of acute pancreatitis. Gut 2005;54(Suppl 3):iii1-9.

50. Jouve P, Bazin JE, Petit A, et al. Epidural versus continuous preperitoneal analgesia during fast-track open colorectal surgery: a randomized controlled trial. Anesthesiology 2013;118:622-30.

51. Harris PA, Taylor R, Thielke R, et al. Research electronic data capture (REDCap)--a metadata-driven methodology and workflow process for providing translational research informatics support. J Biomed Inform 2009;42:377-81.

52. Sauder P, Andreoletti M, Cambonie G, et al. Sédation-analgésie en réanimation (nouveau-né exclu). Annales Françaises d'Anesthésie et de Réanimation 2008;27:541-51.

53. Richards ER, Kabir SI, McNaught CE, et al. Effect of thoracic epidural anaesthesia on splanchnic blood flow. Br J Surg 2013;100:316-21.

54. Freise $\mathrm{H}$, Van Aken HK. Risks and benefits of thoracic epidural anaesthesia. Br J Anaesth 2011;107:859-68.

55. Low JH, Jhs L. Survey of epidural analgesia management in general intensive care units in England. Acta Anaesthesiol Scand 2002;46:799-805. 
Correction: Epidural analgesia in critically ill patients with acute pancreatitis: the multicentre randomised controlled EPIPAN study protocol

Bulyez S, Pereira B, Caumon E, et al. Epidural analgesia in critically ill patients with acute pancreatitis: the multicentre randomised controlled EPIPAN study protocol. BMJ Open 2017;7:e015280. doi: 10.1136/bmjopen-2016-015280.

The list of Collaborators (EPIPAN study group) was missed off the article. This list should read:

Collaborators Sophie Kauffmann, Florian Grimaldi, Charlotte Fernandez-Canal, Philippe Chevaldonné, Julien Pascal, Renaud Guérin, Sébastien Perbet, Sophie Cayot, Thomas Godet, Sophiano Radji, Camille Verlhac, Sandrine Thibault, Christine Rolhion, Justine Bourdier, Emmanuel Futier, Jean-Etienne Bazin, Laetitia Sturma (CHU Clermont-Ferrand, Clermont-Ferrand, France); Lydie Marie-Anne, Olivier Windisch (Geneva university hospitals, Geneva, Switzerland); Annick Puchois, Cyril Boronad, Marine Agullo (Cannes general hospital, Cannes, France); Boris Jung, Gérald Chanques, Cécile Spirito, Albert Prades, Cisse Moussa, Anne Verchere, Claudine Gniadek, Fouad Belafia, Daniel Verzilli, Julie Carr, Audrey De Jong, Yannael Coisel, Jean-Marc Delay, Matthieu Conseil, Marie Gonzalez, Delphine Rosant (Montpellier university hospital, Montpellier, France); Michel Prevot (Nancy university hospital, Nancy, France); Bernard Claud, François Brenas, Lassane Zanre, Phillipe Bray, Hélène Riera, Emilie Gadea-Deschamps (Emile-Roux general hospital, Le Puy-en-Velay, France); David-Paul De Brauwere, Serge Lumbroso, Amélie Maurin, Sophie Lloret, Laurent Muller, Claire Roger, Loubna Elotmani, Audrey Ayral (Nîmes university hospital, Nîmes, France); Suzanne Renard (Saint Luc university hospital, Université Catholique de Louvain, Brussels, Belgium); Nadège Bouskila (Nice Archet 2 university hospital, Nice, France); Gaspard Beaune, Magali Farines-Raffoul, Marie Lebouc (Annecy Genevois general hospital, Annecy, France), on behalf of the EPIPAN study group.

Open Access This is an Open Access article distributed in accordance with the Creative Commons Attribution Non Commercial (CC BY-NC 4.0) license, which permits others to distribute, remix, adapt, build upon this work non-commercially, and license their derivative works on different terms, provided the original work is properly cited and the use is non-commercial. See:http://creativecommons.org/licenses/by-nc/4.0/

C Article author(s) (or their employer(s) unless otherwise stated in the text of the article) 2018. All rights reserved. No commercial use is permitted unless otherwise expressly granted.

BMJ Open 2018;8:e015280corr1. doi:10.1136/bmjopen-2016-015280corr1

Check for updates 